\title{
III. Opposition und Widerstand
}

Wie schon angeführt, brachte Schauff seine Betrachtungen über das Ende der Weimarer Republik im Dezember 1934 zu Papier, während seiner Rückreise von Südamerika auf der „Oceania“, einer der Reisen zur Vorbereitung der Emigration. Der Blick zurück im Zorn wurde zusätzlich durch die Erkenntnis verdunkelt, daß inzwischen alle Hoffnung auf Widerstand und einen Sturz Hitlers geschwunden war.

Schauff war selbst involviert in jenen Widerstandsversuch von konservativer Seite, den Hitler mit der Mordaktion vom 30. Juni 1934 im Zusammenhang mit dem „Röhm-Putsch“ gleichfalls auszuschalten trachtete. Als junger Politiker eher dem linken Zentrum um Wirth zuzurechnen, war Schauff seiner eigenen Aussage nach durch die Erfahrung der Regierung Brüning und aufgrund der allgemeinen politischen Entwicklung am Ende der Weimarer Republik zum Anhänger eines „konservativ-autoritären Regierungssystems“ geworden¹. Im Mai 1933 war er von seinen Ämtern in der Leitung der Reichsstelle für Siedlerberatung und als geschäftsführendes Mitglied der GFK zurückgetreten; bereits einen Monat zuvor war der Verwaltungsrat der Deutschen Siedlungsbank, dem Schauff ebenfalls angehörte, aufgelöst worden ${ }^{2}$. Schauffs Rücktrittsentscheidung wurde offenbar getroffen, um einer drohenden Entlassung mit unwägbaren politischen und finanziellen Komplikationen zuvorzukommen. Sie sollte aber auch den nötigen Spielraum für Widerstand oder die möglicherweise unausweichlich gewordene Emigration schaffen.

Nach Hitlers Ernennung zum Reichskanzler erlebte der Preußens Gloria so skeptisch gegenüberstehende Rheinländer Schauff den „Tag von Potsdam“ (21. März 1933) mit dem Bild des Händedrucks zwischen dem zum Reichskanzler avancierten Weltkriegsgefreiten und dem Feldmarschall und Reichspräsidenten Hindenburg. Er mußte erleben, wie katholische Abgeordnete der NSDAP, darunter Heinrich Himmler, sich im Gottesdienst in der Stadtkirche provokativ respektlos verhielten; die Zentrumsabgeordneten waren zudem bei ihrer Anreise im Autobus nach Potsdam von der Kriminalpolizei molestiert worden, die sie auf Waffen durchsuchen wollte ${ }^{3}$. Dies waren systematische Einschüchterungsversuche, ebenso wie zwei Tage später bei der parlamentarischen Behandlung des Ermächtigungsgesetzes die massive Präsenz der SA im Sitzungssaal. Die demokratischen Parteien resignierten, doch regte sich auch Widerstand.

1 Schauff, Aus meiner beruflichen und politischen Arbeit, S. 3.

2 Schreiben des Stellvertretenden Vorsitzenden des Verwaltungsrates der Deutschen Siedlungsbank an Johannes Schauff vom 13. April 1933 sowie der Gesellschaft zur Förderung der inneren Kolonisation an Johannes Schauff vom 1. Juni 1933 (IfZ, NL Schauff, Bd. 9).

3 Vgl. Morsey, Untergang, S. 128; Bericht des damaligen DNVP-Abgeordneten Edmund Forschbach: „Vier Tage, die Deutschland zum Verhängnis wurden. Meine Erlebnisse und Beobachtungen in Berlin und Potsdam vom 20. bis 23. März 1933“ (IfZ, NL Schauff, Bd. 2). 
Am Abend der pompösen Inszenierung des Potsdamer Staatsaktes wurde Johannes Schauff von seinem bereits erwähnten Reichstagskollegen Hermann Joseph Schmitt, Generalsekretär der katholischen Arbeiter- und Arbeiterinnenvereine, zu einem Treffen mit Edgar Julius Jung im Hotel „Excelsior" mitgenommen. Schmitt hatte sich aufgrund der unwürdigen Behandlung der Zentrumsabgeordneten geweigert, an der Potsdamer Zeremonie teilzunehmen; das Zusammentreffen mit Jung und seinem Kreis war der erste Kontakt mit einem Kreis, der zu aktivem Widerstand bereit war.

Jung, ein aus der Pfalz stammender Münchner Rechtsanwalt, der früher gegen die Separatisten in seiner Heimat gekämpft hatte, war einer der intellektuellen Protagonisten der „konservativen Revolution“4. Sein Einfluß wirkte vor allem in der Studentenschaft, wo er im „Jungakademischen Klub“ in München gegen die nationalsozialistische Bewegung in früher Erkenntnis ihres totalitären politischen Anspruchs Front machte 5 . Dabei entwickelte sich sein politisches Credo in der Auseinandersetzung mit den Nationalsozialisten zu einem dezidiert christlichen Staatsverständnis mit einem transzendentalen Bezug des politischen Handelns. Er wandte sich gegen die Vermassung und Gleichschaltung im totalen Staat und seine Darstellung als Religionsersatz. Gegen Aufklärung und Säkularisierung wurde die Forderung nach einer Rückkehr zu neuer Religiosität und einer universalistischen Weltanschauung erhoben 6 . Jungs Einfluß nahm schließlich noch zu, als er nach Papens Ernennung zum Reichskanzler Juni 1932 als dessen Referent und Ghostwriter in Berlin arbeitete. Er behielt diese Stellung auch nach Papens Rücktritt und gehörte nach dessen Eintritt in die Regierung Hitler zum Mitarbeiterstab der Vizekanzlei. Hier hielt er Kontakte zur Reichswehr und erörterte Möglichkeiten der Opposition u.a. mit Otto Graf, Rudolf Pechel, Martin Spahn, Eduard Stadtler, Hans Bernd Gisevius ${ }^{7}$ und Edmund Forschbach. Letzterer sollte zusam-

4 Zur Biographie Jungs: Forschbach, Edgar J. Jung.; Korrespondenz Schauff - Forschbach, in: IfZ, NL Schauff, Bd. 2 u. 33; Mehringer, Widerstand und Emigration, S. $112 \mathrm{ff}$.

5 Diese Aktivitäten riefen sehr bald die Nationalsozialisten auf den Plan: Vgl. Schreiben Baldur von Schirach an die NS-Hochschulgruppe Jena vom 20. November 1928: „... Dr. Edgar Jung, Halbjude [eine Desinformation], der sich gerne als, hundertprozentiger Revolutionär' bezeichnet, hat mit der NSDAP nichts zu tun. Ich persönlich halte ihn für den schlimmsten Feind der Bewegung" (zit. in: Forschbach, Edgar J. Jung, S. 14).

6 Zur intellektuellen Haltung und Entwicklung Jungs vgl. Forschbach, Edgar J. Jung, und Graß, Edgar Jung, in: Ders., Papenkreis und Röhmkrise, S. 6 ff. und $79 \mathrm{ff}$. Sie steht im geistigen Kontext des konservativen Widerstandes schlechthin. Vgl. Klemperer, Glaube, Religion, Kirche; Solchany, Vom Antimodernismus zum Antitotalitarismus.

7 Pechel, Spahn und Stadtler gehörten zum nationalkonservativen Spektrum der Weimarer Republik: Spahn und Stadtler, beide DNVP, waren 1933 Hospitanten bzw. Mitglieder der NSDAP-Reichstagsfraktion (Stadtler bis November 1933, Spahn bis 1945); Pechel war aufgrund seiner Verbindungen zum konservativen Widerstand 1942-1945 in den Konzentrationslagern Sachsenhausen und Ravensbrück; Gisevius, bis 1935 höherer preußischer Polizeibeamter, gehörte ab 1938 zum aktiven Widerstand (Attentatsvorbereitung), ab 1940 Vizekonsul in Zürich, Kontakte zu Allen W. Dulles und dem amerikanischen Geheimdienst; Otto Graf, MdR Juli 1932-November 1933 (BVP), war Regierungsrat in Amberg und Regensburg, 1933 in Schutzhaft. 
men mit Schmitt und Johannes Schauff die Verbindung mit oppositionellen Kreisen im Rheinland herstellen ${ }^{8}$.

Als Hitler im November 1933 auf einer Einheitsliste den neuen Reichstag wählen ließ, konnten Hindenburg und Papen erreichen, daß eine Anzahl von „parteilosen“ Kandidaten nominiert wurde. Jung und seine Freunde sahen hierin eine Möglichkeit, das NS-Regime von innen zu bekämpfen: Auf der von Jung und Papens Pressereferent Herbert von Bose zusammengestellten Liste figurierten rund 80 Nicht-Nationalsozialisten aus den bürgerlichen Parteien und dem Zentrum, darunter auch Johannes Schauff. Das Unternehmen scheiterte: Nur ein knappes Dutzend der vorgeschlagenen Kandidaten wurde von der Parteikanzlei akzeptiert. Die Ablehnung von Schauff war jedoch schon vorher durch das Büro des Reichspräsidenten erfolgt. Dort hatte man offenbar Schauffs Engagement in der Siedlungsbewegung noch in frischer Erinnerung?.

Nach diesem vergeblichen Versuch einer legalistischen Opposition traten die konspirativen Widerstandspläne dieser Kreise in ein neue Phase. Eine zentrale Rolle spielte dabei die spannungsgeladene Beziehung zwischen Reichswehr und SA, über die Papen und seine jungkonservativen Ratgeber erwartungsgemäß gut unterrichtet waren. Bei einer Zuspitzung der Krise sollte Papen aufgrund seiner Vertrauensstellung beim Reichspräsidenten eine Ablösung der Regierung Hitler erreichen.

Eine bewußte Provokation der Nationalsozialisten bedeutete schließlich Papens Rede vor Marburger Studenten am 17. Juni 1934, die dem konservativen Widerstand zur Artikulation verhelfen sollte. Autor der Rede war Edgar Jung10, der in seinem Redeentwurf noch einmal seine Einschätzung des Nationalsozialismus akzentuierte. Die Rede forderte eine Abwendung vom "Staatstotalismus" und die definitive Einstellung des Terrorregimes und griff die NSDAP wegen ihres Byzantinismus und Personenkults sowie der sich zunehmend ausbreitenden Korruption an. Sie kritisierte die Gleichschaltung der Presse und wandte sich gegen die Ausgrenzung von Minderheiten. Einen „widernatürlichen Totalitätsanspruch" müsse man aus religiöser und christlicher Motivation ablehnen, sonst bestehe die Gefahr, daß Deutschland im Kreis der christlichen Völker Europas isoliert werde. Den Angriffen des Nationalsozialismus auf die Kirchen wurde die Forderung entgegengestellt, auf die Revolution von 1789 und den Vorgang der Säkularisation und Rationalisation einen religiös fundierten Staat folgen zu lassen, der das „Leben wieder unter die natürlichen Gesetze der Schöpfung stellt“. Die neuen Machthaber glaubten, alles Leben organisieren zu können, das damit jedoch nur mechanisiert werde. Dieser hier erkennbare Primat des Geistes über das

8 Edmund Forschbach (1903-1988) war damals Rechtsanwalt in Dortmund, ab 1934 beim Oberlandesgericht in Köln, 1933 (bis Oktober) MdL Preußen für die DNVP; Juli 1933 zum Führer des Cartellverbandes der Katholischen Deutschen Studenten-Verbindungen (CV) berufen, der im März 1934 gleichgeschaltet wurde (Überführung in die „Deutsche Studentenschaft" - siehe unten, S. $68 \mathrm{ff}$.).

9 Vgl. Edmund Forschbach, Edgar Jung und der Widerstand gegen Hitler, S. 84 f.; Forschbach, Brief an Schauff (IfZ, NL Schauff, Bd. 2 u. 33).

10 Abdruck in Forschbach, Edgar J. Jung, S. 154-194; Graß, Edgar Jung, in: Papenkreis, S. $226 \mathrm{ff}$. 
Materielle, der Verlust des Glaubens und die Abwendung von Gott als die eigentliche Ursache für das Aufkommen des Totalitarismus ist ein wesentlicher $\mathrm{Be}$ standteil der geistigen Identität des konservativen Widerstandes, der auch die Frauen und Männer des 20. Juli 1944 prägte ${ }^{11}$. Einige von ihnen gehörten bereits in der beschriebenen Zeitphase zum Widerstand und bildeten über die Jahre hinweg ein Netzwerk, das schließlich, so wird zu zeigen sein, über 1945 hinaus wirksam wurde.

Johannes Schauff hatte bereits Mitte Mai von Edgar Jung einen Entwurf seiner Redevorlage für Papen erhalten und an Brüning weitergeleitet ${ }^{12}$. Allerdings führten die Folgen der Marburger Rede schließlich zu einem ersten Scheitern des konservativen Widerstands. Doch waren dessen Protagonisten bis zum sogenannten Röhm-Putsch, d.h. jener Mordaktion des 30. Juni 1934, durchaus noch vom Gelingen ihrer Pläne überzeugt. Die Marburger Rede hatte in diesen Plänen die taktische Funktion, den Ausbruch der schwelenden Krise zwischen Reichswehr und SA zu beschleunigen. Zwar suchte Goebbels die Verbreitung der Rede in der Presse und im Rundfunk zu unterbinden, die Resonanz in der Öffentlichkeit war dennoch beträchtlich, zumal man der Auslandspresse den Redetext im vorhinein zugespielt hatte ${ }^{13}$.

Papens Mitarbeiter aus der Vizekanzlei versuchten ihrerseits direkten Einfluß auf die Reichswehr zu nehmen und die Opposition gegen die SA zu munitionieren $^{14}$. Die sich zuspitzende Krise sollte zum auslösenden Faktor für die Entlassung der Regierung Hitler und die Übernahme der Macht durch Konservative und Reichswehr werden. Eine zentrale Funktion in diesem Plan kam dem Reichspräsidenten zu, dem die Reichswehr unterstand und der allein die amtierende Regierung entlassen konnte. Die Bemühungen, den Reichspräsidenten durch Vermittlung seines Sohnes Oskar für das weitere Vorgehen zu gewinnen, scheiterten jedoch daran, daß dieser den Umsturzplan im Reichswehrministerium Blomberg mitteilte, der seinerseits Reichenau informierte. Dadurch wurde auch Hitler zumindest soweit ins Bild gesetzt, daß er die ihm von konservativer Seite drohende Gefahr erkannte und - offenbar erst danach - den geplanten Schlag gegen die SAFührung auf den Papen-Kreis und auf weitere katholische und demokratische Politiker ausdehnte ${ }^{15}$. Bereits am 25. Juni 1934 war Edgar Jung aufgrund der von ihm verfaßten Rede auf persönliche Anordnung Hitlers von der Gestapo verhaftet

11 Solchany, Vom Antimodernismus zum Antitotalitarismus, S. $382 \mathrm{ff} .:$ Mehringer, Widerstand und Emigration, S. $187 \mathrm{ff}$.

12 Der Entwurf des Redetextes wurde Schauff im Hause von Wilhelm Fonk (MdR des Zentrums 1932/33, Parteisekretär) im Beisein von Pater Friedrich Muckermann übergeben. Schauff berichtet, daß er selbst die Bekanntschaft von Brüning und Jung vermittelt habe (Schauff an Morsey, 20. Juli 1982, Anlage, IfZ, NL Schauff, Bd. 35).

13 Forschbach, Edgar Jung und der Widerstand gegen Hitler, S. 88; Graß, Edgar Jung, in: Papenkreis, S. $236 \mathrm{ff}$.

14 Die Kontakte bestanden zu den Generälen Rundstedt, Witzleben, Bock, Kluge und Adam (und nicht zu Blomberg und Reichenau) - alles Männer, die später in unterschiedlich enger Verbindung zum militärischen Widerstand gegen Hitler standen; über Jung und Franz Mariaux liefen die Kontakte zu Schleicher und Bredow (Graß, Edgar Jung, in: Papenkreis, S. 235, Anm. 602).

15 Ebenda, S. $264 \mathrm{ff}$. 
worden. Alle Interventionen Papens zu seiner Freilassung waren vergeblich. Fünf Tage nach Jungs Verhaftung, am 30. Juni, fand dann die blutige Generalabrechnung Hitlers mit seinen tatsächlichen und potentiellen Gegnern statt, die, bar jeglicher gesetzlichen Norm, an die Stalinschen Säuberungen erinnert. Neben der SA-Führung und prominenten Parteirivalen wie Gregor Straßer wurden General Kurt von Schleicher ermordet, Edgar Jung, die Katholiken Erich Klausener und Adalbert Probst ${ }^{16}$ sowie der bereits erwähnte Pressechef Papens, Herbert von Bose, Generalmajor Ferdinand von Bredow und Gustav von Kahr ${ }^{17}$.

Schauff war in diese Ereignisse direkt involviert. Als ihn Edmund Forschbach am 25. Juni in Steinfeld in der Eifel besuchte, wohin sich die Familie nach dem Reichstagsbrand zurückgezogen hatte, erörterten sie Pläne zur Ausschaltung der SA und SS im entmilitarisierten Rheinland, wenn die Reichswehr losschlage. Man erwog die Möglichkeit, die St.-Sebastianus-Schützenbruderschaften, die zu diesem Zeitpunkt mehr als 100000 aktive Mitglieder umfaßten ${ }^{18}$, durch die Reichswehr bewaffnen zu lassen ${ }^{19}$. Die Vermittlerrolle zwischen Reichswehr und den St.-Sebastianus-Bruderschaften hatte Schauffs Freund Adalbert Probst übernommen. Das weitere Vorgehen sollte bei einer Zusammenkunft mit Edgar Jung, Forschbach, Schauff und Paul Franken ${ }^{20}$ in Forschbachs Wohnung in Köln abgesprochen werden; Franken wollte zudem Walther Hensel hinzuziehen, damals Syndikus der Stadtverwaltung Düsseldorf ${ }^{21}$. Als dieses Treffen schließlich am 30. Juni stattfand, war Jung allerdings bereits verhaftet und der Widerstand ohne Unterstützung der Reichswehr gescheitert. Über ausländische Sender hörten die Verschwörer von der Ermordung Jungs, Boses und Probsts.

16 Klausener war Ministerialdirektor im Reichsverkehrsministerium, Leiter der Katholischen Aktion in Berlin, einer der einflußreichsten katholischen Laien; Adalbert Probst war Mitglied der Reichsleitung der Deutschen Jugendkraft (DJK), verantwortlich für den - im Zuge der nach 1933 einsetzenden Gleichschaltungsbestrebungen der Nationalsozialisten gegenüber den konfessionellen Jugendverbänden - bei der DJK eingeführten, vormilitärisch ausgerichteten Geländesport (vgl. Schellenberger, Katholische Jugend, S. $134 \mathrm{ff}$.$) .$

17 Unter Kahr - damals Generalstaatskommissar in Bayern - war 1923 der „Hitler-Putsch“ niedergeschlagen worden; Bredow war Chef des Ministeramts und Vertrauter des Reichswehrministers und späteren Reichskanzlers Kurt von Schleicher. Zur Liquidierung der „alten Eliten“ durch das NS-Regime anläßlich des „Röhm-Putsches“ vgl. u.a. Mehringer, Widerstand und Emigration, S. $114 \mathrm{ff}$.

18 Die "Erzbruderschaft vom Heiligen Sebastianus" war vor allem im Rheinland und in Westfalen aktiv und eine typische Organisation des katholischen Milieus, das dem Nationalsozialismus ablehnend gegenüberstand. Sie wurde 1936 aufgelöst. Wiedergründung 1948 auf Diözesanebene und 1951 als „Zentralverband der Historischen Deutschen Schützenbruderschaften“. Die Zahlenangaben nach einer Information des Archivs des Zentralverbands der Historischen Deutschen Schützenbruderschaften in Leverkusen, das über keine Unterlagen mehr verfügt, diese weiter zu präzisieren (Hochrechnung ca. 125000 Mitglieder in den Jahren 1933/34).

19 Forschbach, Brief an Johannes Schauff, S. $82 \mathrm{f}$.

20 Franken war 1930-1936 Geschäftsführer des Kartell-Verbandes der katholischen Studentenvereine Deutschlands, 1937-1939 in Haft.

21 Hensel gehörte zum Führungskreis der katholischen Jugend Deutschland und stand in Verbindung mit deren Generalpräses Ludwig Wolker (Zeugenschrifttum Walther Hensel, IfZ, ZS 534). 
Der Bauernhof in der Eifel wurde nun zum Refugium nicht nur der Familie Schauff, sondern auch für gefährdete und politisch verfolgte Freunde. Der Eifelhof war das Elternhaus von Karin Schauff, das Ende der zwanziger Jahre in den Besitz der jungen Eheleute gelangt war, zu einer Zeit, als sie, bereits in Unruhe wegen der zunehmenden politischen Radikalisierung, ein sicheres Ausweichquartier suchten ${ }^{22}$. Als einer der ersten fand hier Edmund Forschbach Zuflucht, dem wegen seiner persönlichen Nähe zu Jung politische Verfolgung drohte und der im Juli 1934 auch sein Reichstagsmandat verloren hatte ${ }^{23}$. Über Steinfeld floh Forschbach vorübergehend nach Holland, wo er in dem Jesuitenkolleg in Valkenburg Zuflucht fand ${ }^{24}$.

Als Forschbach im September 1934 wieder zurückkehren konnte, hatte vor allem jener rheinische Widerstandskreis an Kontur gewonnen, dessen Anfänge mit Edgar Jungs Aktivitäten zusammenhängen. Dieser Kreis besaß über Hermann Joseph Schmitt Verbindung zu den katholischen Arbeitervereinen, deren Widerstand sich im Ketteler-Haus in Köln konzentrierte ${ }^{25}$, und zu Vertretern der ehemaligen christlichen Gewerkschaften. Johannes Schauff hatte den Kontakt zu Karl Thieme und mit diesem zusammen zu den katholischen Jungmänner-Organisationen hergestellt. Über den Berliner Rechtsanwalt Josef Wirmer ${ }^{26}$ und über Forschbach waren Walther Hensel und Paul Franken zu dem Kreis um Jung gestoßen. Nach dem 30. Juni 1934 drängten vor allem Männer wie Walther Hensel auf den Aufbau einer planmäßigen Widerstandsbewegung. Zu diesem Kreis gehörten ebenso der ehemalige Landesgeschäftsführer der christlichen Gewerkschaften, Heinrich Körner, sowie Eduard Stadtler. Über Hensel lief der Kontakt $\mathrm{zu} \mathrm{Karl} \mathrm{Arnold}{ }^{27}$, und es bestanden über Franken enge Verbindungen zu Josef Müller ${ }^{28}$ in München. Nach Ostern 1935 fand in der Kölner Wohnung Forsch-

22 Karin Schauff, Das Klingelband ("Der Eifelhof ${ }^{\text {) }), ~ S . ~} 45 \mathrm{f}$.

23 Forschbach war im November 1933 als Hospitant der NSDAP in den Reichstag gewählt worden.

24 Karin Schauff habe ihn zum Bahnhof noch ein Stück des Weges begleitet und ihm Guardinis „Kreuzweg“ mitgegeben: Forschbach, Brief an Schauff, S. 84; Forschbach, Edgar J. Jung ("Auf der Flucht"), S. $130 \mathrm{ff}$.

25 Die Verbandsführung bestand 1933 aus Otto Müller (Verbandsvorsitzender), Bernhard Letterhaus (Verbandssekretär), Joseph Joos und Nikolaus Groß. Im Ketteler-Haus fanden ab Mitte der dreißiger Jahre Zusammenkünfte von christlichen Gewerkschaftern und Zentrumspolitikern statt, von denen einige - Jakob Kaiser, Nikolaus Groß, und Bernhard Letterhaus - über Alfred Delp später in Verbindungen zu Widerstandskreisen im Umfeld des 20. Juli 1944 standen. Groß, Letterhaus und Delp wurden hingerichtet. Vgl. Mehringer, Widerstand und Emigration, S. $100 \mathrm{ff}$.

26 Wirmer gehörte zu den führenden Köpfen der Berliner Zentrumspartei und der katholischen Jugendbewegung (1928 mit Joseph Joos Initiator des Reichsjugendausschusses des Zentrums).

27 Arnold war in der christlichen Gewerkschaftsbewegung tätig; 1947-1956 Ministerpräsident von Nordrhein-Westfalen.

28 Müller war Rechtsanwalt in München, vor 1933 BVP; ab 1939 Anschluß an die Widerstandsbewegung um Ludwig Beck und Wilhelm Canaris; 1946-1949 Landesvorsitzender der CSU, bayerischer Justizminister (1947-1952) und stellvertretender Ministerpräsident (1947-1950). 
bachs ein Treffen statt, an dem Johannes Schauff, Karl Thieme und Karl Fütterer ${ }^{29}$ teilnahmen; Thieme propagierte bei dieser Gelegenheit aktiven Widerstand gegen Hitler, denn dieser strebe nach einer völligen Umwälzung des europäischen Staatensystems, die nur durch einen Krieg zu erreichen $\operatorname{sei}^{30}$.

Weitere Verbindungen bestanden zu dem ehemaligen Düsseldorfer Oberbürgermeister Robert Lehr, in Berlin konnten wenig später Hensel und Körner den Kontakt zu Jakob Kaiser herstellen - und damit eine Verbindung zu einem Kreis in der Hauptstadt, in den auch Nikolaus Groß, Rudolf Pechel, Josef Wirmer und Franz Etzel einbezogen waren ${ }^{31}$. Dort versuchten die Rheinländer weiterhin, die Armee in Pläne zum Sturz des nationalsozialistischen Regimes einzubinden, da man realistischerweise davon ausging, daß nur mit ihrer Unterstützung ein solches Vorhaben gelingen könne. Durch die Vermittlung Lehrs kam schließlich der Kontakt Kaisers zu Generaloberst Hammerstein-Equord, bis 1. Februar 1934 Chef der Heeresleitung und Gegner Hitlers, zustande - und damit eine Verbindung zur Bendlerstraße, die später auch Wilhelm Leuschner und Max Habermann $^{32}$ einschloß und die sich bis zum 20. Juli 1944 als tragfähig erweisen sollte.

Im Rheinland selbst bemühte man sich mit Unterstützung von Theodor Scharmitzel, dem ehemaligen Generalsekretär der Windthorstbunde, um Einfluß auf die katholische Akademikerschaft. In diesem Kreis agierte auch Pater Ignatius Eschmann O. P. , der 1937 mit großer öffentlicher Resonanz gegen die antikirchlichen Kampagnen der Nationalsozialisten vor dem Hintergrund der manipulierten Ordensprozesse von der Kanzel protestierte und infolgedessen am 2. Juli 1937 von der Gestapo verhaftet wurde. Eine weitere Verhaftungswelle hatte als konkreten Auslöser die Aufdeckung von Korruptionsskandalen in Düsseldorf, in die Nationalsozialisten verwickelt waren; ihr fielen Walther Hensel und später auch Paul Franken und Jakob Kaiser zum Opfer ${ }^{33}$.

Im Zusammenhang mit dieser Atmosphäre der zunehmenden Bedrohung und Verfolgung erinnert sich Forschbach "besonders lebhaft" an eine Zusammenkunft mit Schauff am 16. November 1937 in Steinfeld, an der auch der frühere Vertreter der Rheinprovinz im Reichsrat, Wilhelm Hamacher, sowie die Journalisten Bachmann und Kadow teilnahmen. „Unsere Stimmung“, so Forschbach, „war gedrückt, weil am Allerheiligentage Walther Hensel, der sich mutig für einen Aufbau von Widerstandskreisen im Rheinland eingesetzt hatte, von der Gestapo verhaftet worden war. Kurz danach wurden auch Paul Franken und Jakob Kaiser

${ }^{29}$ Fütterer kam wie Schauff aus der Siedlungsbewegung; nach 1945 Direktor der Siedlungsgesellschaft „Rote Erde“ in Münster. Über die oppositionelle Tätigkeit Fütterers unter dem Nationalsozialismus und die Zusammenarbeit mit Schauff vgl. Schauffs Rede zum 60. Geburtstag Fütterers, IfZ, NL Schauff, Bd. 33.

30 Forschbach, Edgar J. Jung, S. 136.

31 Kaiser und Etzel waren später (1949-1957 bzw. 1957-1961) Minister in der Regierung Adenauer.

32 Max Habermann, führender Vertreter des Deutschnationalen Handlungsgehilfen-Verbands, war u. a.Vertrauensmann Brünings (Morsey, Untergang, S. 238, Anm. 16).

33 Hensel wurde am 1. November 1937 verhaftet, Kaiser am 2. Februar 1938; er war bis 27. Oktober 1938 in Haft und stand ab 1941 in Verbindung mit Carl Goerdeler und dem Kreis um den 20. Juli 1944 (Zeugenschriftum Hensel, IfZ, ZS 534; Forschbach, Edgar J. Jung, S. $141 \mathrm{ff}$.). 
eingekerkert. Wir wußten jetzt, daß die Gestapo in unserer unmittelbaren Nähe ihre Opfer aufspürte ... Am Abend dieses Tages gingen wir in die Steinfelder Kirche, um für unsere gefangenen Freunde und für uns selbst den Schutz Gottes anzuflehen." 34

Die Familie Schauff, die unterdessen definitiv nach Steinfeld umgezogen war, nachdem man noch eine Zeitlang zwischen Berlin und der Eifel hin und her gependelt war, geriet allerdings selbst zunehmend ins Visier der Gestapo. Die Kinder - mit der Geburt von Zwillingen in Steinfeld waren es unterdessen sechs wurden in der Schule ausgegrenzt, und die von Karin Schauff bereits bei der Übernahme des Hofes zusammen mit ihrer Schwester Agnes begründete Siedlerinnenschule zur Unterstützung der Arbeit der GFK $^{35}$ sollte gleichgeschaltet werden. Schauff wurde wiederholt von der Gestapo vorgeladen - in Berlin aufgrund seiner Verbindung zu den Religiösen Sozialisten und insbesondere zu Maria Grollmus, in Steinfeld, weil man zu Recht vermutete, daß das Schauffsche Anwesen als Fluchtstation für politisch und rassisch verfolgte Menschen diente. In der Tat waren es nicht wenige, die vor allem von Karin Schauff versorgt und von ortskundigen Freunden über die grüne Grenze gebracht wurden, darunter Waldemar Gurian, ein Berliner Studienfreund der Schauffs ${ }^{36}$. Johannes Schauff, der bereits häufig auf Reisen war, um Emigrationsmöglichkeiten für Regimegegner zu erkunden - dabei gelangte er anläßlich einer Erkundungsreise des Völkerbundes bis in die Mandschurei ${ }^{37}$-, traf sich mit seiner Frau nur noch heimlich und außerhalb des Wohnortes ${ }^{38}$.

${ }^{34}$ Forschbach, Brief an Schauff, S. $84 \mathrm{f}$.

35 Karin Schauff, Zur Geschichte des Hauses Steinfeld (Ms.), S. 4 (IfZ, NL Schauff, Bd. 40).

36 Siehe unten, S. 206 sowie Anm. 17; Karl Thieme berichtet in einer "Erklärung" vom 20. November 1956: Neben Gurian wurde in den Jahren 1935/36 „der einzige Sohn Erik der nach Prag emigrierten Schwiegertochter des Dichters Gustav Falke bei Schauffs aufgenommen; in der Zwischenzeit haben sie noch mehreren anderen Gefährdeten über kürzere oder längere Zeiträume hinweg bei sich Asyl gewährt; auch ich selbst und andere Mitarbeiter von Generalpräses Wolker im Düsseldorfer katholischen Jugendhaus durften wiederholt in der verhältnismäßigen Geborgenheit des Steinfelder Hauses Zuflucht finden ... Karin Schauff hat durch all dies persönlich ein großes Risiko übernommen." (IfZ, NL Schauff, Bd. 8); vgl. auch Heinz Hürten, Waldemar Gurian, S. 89.

37 Die Reise fand 1936 statt, die Rückreise über Kanada und die Vereinigten Staaten (Mitteilung Schauffs an Professor Arnold Wolfers/Yale University vom 10. September 1942). Wolfers war Schauffs Studienkollege an der Berliner Hochschule für Politik gewesen (IfZ, NL Schauff, Bd. 8); ein von Schauff signiertes Photo („Auf Weltreise mit Kiesel Stein“) in IfZ, NL Schauff, Bd. 28.

38 Vgl. Karin Schauff, Das Klingelband, S. $47 \mathrm{ff}$. 